\title{
Heat Transfer Analysis For Oscillating Flow of Magnetized Fluid By Using The Modified Prabhakar- Like Fractional Derivatives
}

\section{Ali Raza}

University of Engineering and Technology

\section{Sami Ullah Khan}

COMSATS University Islamabad

M. Ijaz Khan ( $\square$ mikhan@math.qau.edu.pk)

Riphah International University l-14

\section{Essam Roshdy El-Zahar}

Prince Sattam Bin Abdulaziz University

\section{Research Article}

Keywords: Heat transfer, MHD flow, oscillating flow, Prabhakar-like fractional derivatives

Posted Date: December 1st, 2021

DOI: https://doi.org/10.21203/rs.3.rs-1086428/v1

License: @ (i) This work is licensed under a Creative Commons Attribution 4.0 International License. Read Full License 


\title{
Heat transfer analysis for oscillating flow of magnetized fluid by using the modified Prabhakar-like fractional derivatives
}

\author{
Ali Raza ${ }^{1}$, Sami Ullah Khan ${ }^{2}$, M. Ijaz Khan ${ }^{3}$, Essam Roshdy El-Zahar ${ }^{4,5, *}$ \\ ${ }^{1}$ Department of Mathematics, University of Engineering and Technology, Lahore 54890, \\ Pakistan \\ ${ }^{2}$ Department of Mathematics, COMSATS University Islamabad, Sahiwal 57000, Pakistan \\ ${ }^{3}$ Department of Mathematics and Statistics, Riphah International University I-14, Islamabad \\ 44000, Pakistan \\ ${ }^{4}$ Department of Mathematics, College of Science and Humanities in Al-Kharj, Prince Sattam \\ Bin Abdulaziz University, P.O. Box 83, Al-Kharj 11942, Saudi Arabia \\ ${ }^{5}$ Department of Basic Engineering Science, Faculty of Engineering, Menoufia University, \\ Shebin El-Kom 32511, Egypt \\ (*Correspondence: mikhan@ math.qau.edu.pk (M. I. Khan))
}

\begin{abstract}
:
In this analysis, an unsteady and incompressible flow of magnetized fluid in presence of heat transfer has been presented with fractional simulations. The oscillating plate with periodically variation has induced the flow. The model is formulated in terms of partial differential equations (PDE's). The traditional PDEs cannot analyze and examine the physical behavior of flow parameters with memory effects. On this end, the solution approach is followed with the efficient mathematical fractional technique namely Prabhakar fractional derivative. The nondimensional leading equations are transformed into the fractional model and then solved with the help of the Laplace transformation scheme. The effects and behavior of significant physical and fractional parameters are analyzed graphically and numerically. As a result, we have concluded that the temperature and velocity profiles decrease with the enhancement of fractional parameters. Furthermore, with time both (temperature and velocity fields)decreasing away from the plate and asymptotically increases along $y$-direction, which also satisfies the corresponding conditions.
\end{abstract}

Keywords: Heat transfer, MHD flow, oscillating flow, Prabhakar-like fractional derivatives.

\section{Introduction}

The investigations regarding the heat transfer phenomenon is important because all the industrial mechanism and engineering processes involves this phenomenon. The key importance of heat transfer is inspected in the thermal systems, nuclear processes, gas processing, various chemical processes, refining industries, electronics etc. This chemical 
processes and processing industries also involves the heat transfer significances like cooling and heating of different chemical equipment and production of chemical materials. The modeling of heat transfer is based on the famous Fourier's theory. Following to such novel implications in mind, a variety of research has been done by investigators. Turkyilmazoglu [1] inspected the heat transfer fluctuation for the viscoelastic fluid with distinct types of physical properties. Mishra and Bhatti [2] observed the influences of Ohmic heating for the convectively heated problem via numerical simulations. Nayak et al. [3] presented the heat transfer investigation for the magnetized material subject to the saturated porous space. Alamri et al. [4] presented the novel thermal contribution regarding the heat transfer phenomenon in stretched cylinder. The vertical flow determination for the heat transfer problem with modified Fourier's expressions has been worked out by $\mathrm{Li}$ et al. [5]. Waini et al. [6] focused on the participation of hybrid nanoparticles for improving the heat characteristics against the shear flow. Hayat et al. [7] utilized the melting heating applications for the variable thickness flow subject to the chemical aspect. Hassan et al. [8] observed the heat transfer rate for the shear thinning type nano-materials with hybrid nanoparticles with experimental justifications. The heat pattern in the horizontally moving space with radiative phenomenon was identified by Benos et al. [9]. Mahanthesh et al. [10] presented the heat transfer analysis for the isothermal wedge flow with characterization of $\mathrm{Ag}$ and $\mathrm{MoS}_{2}$ nanoparticles. Shehzad et al. [11] performed a computation thermal problem focusing the Ohmic and dissipation features. Khan et al. [12] provided the improved heat transfer mechanism based on the slip flow with micropolar nanofluid and thermal radiative approach.

The progress in the fractional

The growing work in the fractional calculus provides modern definitions of various kinds of derivatives and integrals. Due to new characterization of operators, the interest is developed by scientists towards the different types of fractional derivatives. In the world real problems, many fractional techniques are implemented like diffusion problems, thermal modeling, biological problems, plasma physics, bio-chemistry, industrial problem and engineering. In the fractional derivatives, the descriptions of non-local and local definitions are quite interesting. Amongst both, the non-local derivatives are assumed to be more novel as these derivatives convey the history of function. The concept of fractional simulations is completed with the different types of functions and definitions. One of the most famous fractional research involving the fractional derivatives was reported by Caputo and Fabrizio [13]. This research successfully explained the aspects of non-local kernels and phenomenon. Harrouche et al. [14] implemented the approach of Caputo-Fabrizio (CF) derivatives for performing the simulations for the drug 
pharmacokinetic problem. Moore et al. [15] used the Caputo and Fabrizio model for the treatment of HIV/AIDS disease problem. The implementations of Caputo-Fabrizio derivatives simulations regarding the diffusion flow problem were worked out by Wei et al. [16]. Alzahrani et al. [17] used the CF- definition for the coronavirus modeling problem. Momani et al. [18] focused on the solution of Bernoulli equation via CF model. The work of Shaikh et al. [19] explained the physical insight of reactive diffusion thermal model where the simulations were carried out by $\mathrm{CF}$ approach. The integral approach for the Hermite-Hadamard inequalities with new convex function with help of CF-model has been focused by Wang et al. [20]. The study of Covid-19 diseases with CF implementation was performed by Baleanu et al. [21]. In recent times, some modifications has been done by researchers regarding the CF definition. On this end, Atangana and Baleanu (AB) [22] defined new operators and definitions of fractional simulations known as $\mathrm{AB}$-derivatives. The $\mathrm{AB}$-derivatives is defined on the basis of nonsingular kernels which terms as Mittag-Leffler functions. This definition is proved more effective as it does not allow any singularity. While inspecting the comparative task between $\mathrm{CF}$ and $\mathrm{AB}$-fractional definitions, the $\mathrm{AB}$ derivatives are declared more precise. Sheikh et al. [23] used the AB- fractional technique for the solution of Casson fluid model problem with external chemical reaction assertive. Panda et al. [24] focused on the implementation of same approach for a fractional type Willis aneurysm problem. Moreover, the discussion for the perturbed boundary value system with nonlinear singularity was also provided. Kumar et al. [25] observed the infection and threats of mosaic disease via mathematical modeling and later on fractional approach with AB-deviations was followed for the simulation procedure. Raza et al. [26] used same definitions for assessing the nanoparticles characterization over vertically infinite plate. The AB-formulation for the integro-differential systems was intended by Arjunan et al. [27]. Kumar et al. [26] used the CF and AB-fractional work for the mathematical formulation of a mosaic disease problem. Some more recent work can be seen in refs. [27-32]. This fractional investigation deals with the determination of heat transfer applications for the viscous fluid due to periodically accelerating surface. The applications of inclined magnetic force are also implemented. The model problem is solved with the latest frame work of fractional derivatives namely Prabhakar fractional approach. The results are simulated at various time instant due to periodic oscillation. The physical insight parameters are addressed.

\section{Problem description}

Consider an unsteady compressible fluid is moving on an infinite inclined plane with the inclination of angle $\delta$ by variable temperature. It also supposed a magnetic field of strength $B_{o}$ is applied on the plate in the absence of any electric field. Initially at $t=0$, the plate and fluid 
both are in the rest position with constant temperature $T_{\infty}$ (see Fig. 1). With time $t>0^{+}$the plate starts to oscillate with constant velocity $U_{o} H(t) \cos (\omega t)$, where $\omega$ representing the frequency of oscillation, and the fluid starts to flow on the plate due to oscillations. So with the help of Boussinesq's approximation, the partial differential leading equations that govern this problem can be stated as:

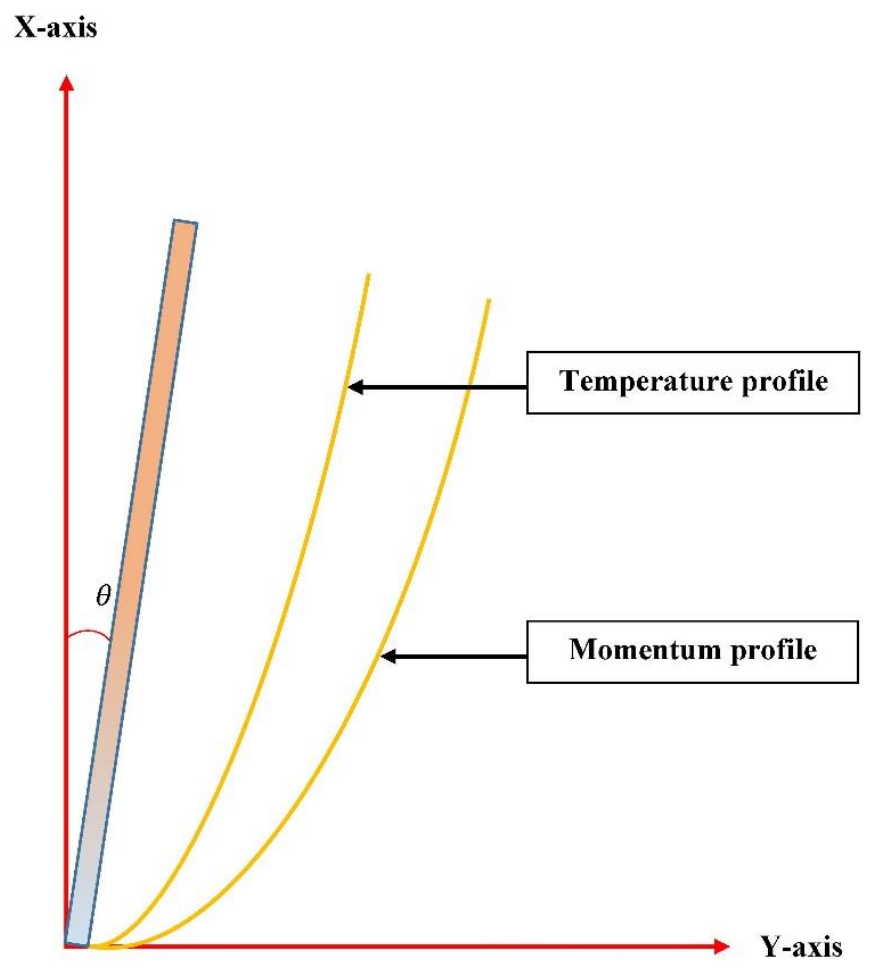

Fig 1: Flow geometry

$\rho \frac{\partial w_{(\zeta, t)}}{\partial t}=\mu \frac{\partial^{2} w_{(\zeta, t)}}{\partial \zeta^{2}}-\frac{\sigma B_{o}^{2}}{\rho} \sin (\theta) w_{(\zeta, t)}+g \beta_{T}\left(T_{(\zeta, t)}-T_{\infty}\right) \operatorname{Cos}(\delta)$

$\rho C_{p} \frac{\partial T_{(\zeta, t)}}{\partial t}=-\frac{\partial q_{(\zeta, t)}}{\partial \zeta}-Q\left(T_{(\zeta, t)}-T_{\infty}\right)$

$q_{(\zeta, t)}=-k \frac{\partial T_{(\zeta, t)}}{\partial \zeta}$

where $q_{(\zeta, t)}$ signifies the thermal flux rate by Fourier's law with its consistent boundary conditions, as follows

$$
\begin{array}{lrl}
w_{(\zeta, 0)} & =0, \quad T_{(\zeta, 0)}=T_{\infty}, & ; \quad \zeta \geq 0 \\
w_{(0, t)}=U_{o} H(t) \cos (\omega t), & T_{(0, t)}=T_{\infty}+\left(T_{w}-T_{\infty}\right), & t>0 \\
w_{(\zeta, t)} \rightarrow 0, & T_{(\zeta, t)} \rightarrow T_{\infty}, \quad \text { as } \quad \zeta \rightarrow \infty
\end{array}
$$

For the non-dimensional for of the leading equations and consistent conditions, introducing the following non-dimensional values 


$$
w^{*}=\frac{w}{U_{o}}, \quad \zeta^{*}=\frac{U_{o}}{v_{f}} \zeta, \quad t^{*}=\frac{U_{o}^{2}}{v_{f}} t, \quad T^{*}=\frac{T_{(\zeta, t)}-T_{\infty}}{T_{w}-T_{\infty}}, \quad q^{*}=\frac{q}{q_{o}}
$$

into the proceeding governing equations and conditions (1)-(7) and neglecting the steric notation. We obtain the subsequent non-dimensional forms as follows

$\frac{\partial w_{(\zeta, t)}}{\partial t}=\Omega_{1} b_{1} \frac{\partial^{2} w_{(\zeta, t)}}{\partial \zeta^{2}}-M \sin (\theta) w_{(\zeta, t)}+G r T_{(\zeta, t)} \operatorname{Cos}(\delta)$

$\frac{\partial T_{(\zeta, t)}}{\partial t}=-\Omega_{2} \frac{\partial q_{(\zeta, t)}}{\partial \zeta}-F T_{(\zeta, t)}$

$q_{(\zeta, t)}=-c_{1} \frac{\partial \vartheta_{(\zeta, t)}}{\partial y}$

withthe following non-dimensional conditions

$$
\begin{gathered}
w_{(\zeta, 0)}=0, \quad T_{(\zeta, 0)}=0 ; \quad \zeta \geq 0 \\
w_{(0, t)}=H(t) \cos (\omega t), \quad T_{(0, t)}=1, \quad t>0 \\
w_{(\zeta, t)} \rightarrow 0, \quad T_{(\zeta, t)} \rightarrow 0 ; \quad \zeta \rightarrow \infty
\end{gathered}
$$

Where:

$$
\begin{gathered}
\Omega_{1}=\frac{\tau_{o}}{\rho U_{o}^{2}}, \quad \Omega_{2}=\frac{q_{o}}{\rho C_{p}\left(\vartheta_{w}-\vartheta_{\infty}\right) U_{o}}, \quad \mathrm{~b}_{1}=\frac{\mu U_{o}^{2}}{v \tau_{o}}, \quad \mathrm{c}_{1}=\frac{k U_{o}\left(\vartheta_{w}-\vartheta_{\infty}\right)}{v q_{o}} \\
F=\frac{Q v}{\rho C_{p} U_{o}^{2}}, \operatorname{Pr}=\frac{\mu C_{p}}{\kappa}, \quad G r=\frac{g\left(v \beta_{T}\right)_{f}\left(\vartheta_{w}-\vartheta_{\infty}\right)}{U_{o}^{3}}
\end{gathered}
$$

Here for the solution of momentum and energy equations we have exploited an efficient fractional mathematical model well known as Prabhakar fractional derivative, which can be defined mathematically as $[33,34]$ :

$$
\begin{gathered}
C_{\mathfrak{D}_{\alpha, \beta, \alpha}^{\gamma}}^{\gamma} h(t)=E_{\alpha, n,-\beta, \alpha}^{-\gamma} h^{n}(t)=e_{\alpha, n,-\beta}^{-\gamma}(\alpha ; t) * h^{n}(t) \\
=\int_{0}^{t}(t-\tau)^{n-\beta-1} E_{\alpha, n,-\beta}^{-\gamma}\left(\alpha(t-\tau)^{\alpha}\right) h^{n}(\tau) d \tau
\end{gathered}
$$

Where ${ }^{C} \mathfrak{D}_{\alpha, \beta, \alpha}^{\gamma} h^{(n)}$ represents thePrabhakar fractional operator and the nth derivative of $h(t) \in A C^{n}(0, b), A C^{n}(0, b)$ respectively, and

$$
E_{\alpha, \beta, \alpha}^{\gamma} h(t)=\int_{0}^{t}(t-\tau)^{\beta-1} E_{\alpha, \beta}^{-\gamma}\left(\alpha(t-\tau)^{\alpha}\right) h(\tau) d \tau
$$

is known as Prabhakar integral with

$$
E_{\alpha, \beta}^{\gamma}(z)=\sum_{m=0}^{\infty} \frac{\Gamma(\gamma+m) z^{m}}{m ! \Gamma(\gamma) \Gamma(\alpha m+\beta)}
$$


is of three-parametric Mittage-Leffer function, and $e_{\alpha, \beta}^{\gamma}(\alpha ; t)=t^{\beta-1} E_{\alpha, \beta}^{\gamma}\left(\alpha t^{\alpha}\right)$ is the Prabhakar kernel. The Laplace transform of Prabhakar fractional derivative operator ${ }^{C} \mathfrak{D}_{\alpha, \beta, \alpha}^{\gamma}$ is derived as

$$
\begin{gathered}
\mathcal{L}\left[{ }^{C} \mathfrak{D}_{\alpha, \beta, \alpha}^{\gamma} \mathrm{h}(t)\right]=\mathcal{L}\left[h^{m}(t) * e_{\alpha, m-\beta}^{-\gamma}(\alpha ; t)\right]=\mathcal{L}\left\{h^{m}(t)\right\} \mathcal{L}\left\{e_{\alpha, m-\beta}^{-\gamma}(\alpha ; t)\right\} \\
=\mathcal{L}\left\{h^{m}(t)\right\} s^{\beta-m}\left(1-\alpha s^{-\alpha}\right)^{\gamma}
\end{gathered}
$$

and by taking $\beta=\gamma=0$, we can obtain the classical Fourier's law. And as the Prabhakar fractional derivative mostly depends on Fourier's law of thermal conductivity, so the Fourier's law in the sense of Prabhakar fractional derivative will become as,

$$
q_{(\zeta, t)}=-c_{1}{ }^{t} \mathfrak{D}_{\alpha, \beta, \alpha}^{-\gamma} \frac{\partial T_{(\zeta, t)}}{\partial \zeta}
$$

\section{Solution of the problem}

\subsection{Solution of the energy field}

As the energy equation involve Fourier's law of thermal flux so, by utilizing the LT scheme on Eqs. (8) and (9) for the solution of energy profile and on its corresponding conditions

$$
\begin{aligned}
& \mathrm{s} \bar{T}_{(\zeta, s)}=-\Omega_{2} \frac{\partial \bar{q}_{(\zeta, s)}}{\partial \zeta}-F \bar{T}_{(\zeta, s)} \\
& \bar{q}_{(\zeta, s)}=-c_{1}\left(s^{\beta}\left(1-\alpha s^{-\alpha}\right)^{\gamma}\right) \frac{\partial \bar{T}_{(\zeta, s)}}{\partial \zeta} \\
& \bar{T}_{(0, s)}=\frac{1}{s}, \bar{T}_{(\zeta, s)} \rightarrow 0 ; \quad \zeta \rightarrow \infty
\end{aligned}
$$

Introducing Eq. (13) into (12), for the solution of temperature field

$$
\frac{\partial^{2} \bar{T}_{(\zeta, s)}}{\partial \zeta^{2}}-\frac{1}{\Omega_{2} c_{1}} \frac{F+s}{s^{\beta}\left(1-\alpha s^{-\alpha}\right)^{\gamma}} \bar{T}_{(\zeta, s)}=0
$$

By using the above conditions and solving the ordinary differential Eq. (15), the solution of temperature distribution will be yield as

$$
\bar{T}_{(\zeta, s)}=\frac{1}{s} e^{-\zeta \sqrt{\frac{1}{\Omega_{2} c_{1 s} \beta\left(1-\alpha s^{-\alpha}\right) \gamma}}}
$$

For the inverse of Laplace of Eq. (16), we will use numerical techniques namely as Stehfest and Tzou's algorithms in Table 1.

\subsection{Classical solution of Temperature Field $(\beta=\gamma=0)$}

For classical thermal transport take $\beta=\gamma=0$, so

$$
\mathcal{L}\left[e_{\alpha, 0}^{0}(\alpha ; t)\right]=1=\delta(t)
$$

Where $\delta(t)$ is the Dirac's Delta distribution. By using this, the generalized Fourier's law will be transformed into classical Fourier's law. And

$$
\bar{T}_{(\zeta, s)}=\frac{1}{s} e^{-\zeta \sqrt{\frac{1}{\Omega_{2} c_{1}}(F+s)}}
$$


With its Laplace inverse

$$
T_{(\zeta, t)}=\frac{1}{2} e^{-\zeta \sqrt{\frac{F}{\Omega_{2} \mathrm{c}_{1}}}}\left(2-\operatorname{Erfc}\left[\frac{2 \sqrt{\Omega_{2} \mathrm{c}_{1} F} t-\zeta}{2 \sqrt{\Omega_{2} \mathrm{c}_{1} t}}\right]+e^{2 \zeta \sqrt{\frac{F}{\Omega_{2} \mathrm{c}_{1}}}} \operatorname{Erfc}\left[\frac{2 \sqrt{\Omega_{2} \mathrm{c}_{1} F} t+\zeta}{2 \sqrt{\Omega_{2} \mathrm{c}_{1} t}}\right]\right)
$$

\subsection{Solution of the momentum field}

In this section, the solution of the momentum equation will be derived with the same procedure as used for the energy equation solution. By utilizingthe LTscheme on Eq. (7) and using its corresponding conditions, we obtain

$$
\begin{aligned}
s \bar{w}_{(\zeta, s)}=\Omega_{1} \mathrm{~b}_{1} \frac{\partial^{2} w_{(\zeta, s)}}{\partial \zeta^{2}}-M \sin (\theta) \bar{w}_{(\zeta, s)}+G r \bar{T}_{(\zeta, s)} \operatorname{Cos}(\delta) \\
\bar{w}_{(0, s)}-\frac{s}{s^{2}+\omega^{2}}=0, \quad \bar{w}_{(\zeta, s)} \rightarrow 0 ; \quad \zeta \rightarrow \infty
\end{aligned}
$$

Using these conditions, the solution of the momentum equation will become as

$$
\begin{gathered}
\bar{v}_{(y, s)}=\frac{s}{s^{2}+\omega^{2}} e^{-y \sqrt{\frac{s+M}{\Omega_{1} \mathrm{~b}_{1}}}} \\
+\frac{G r \cos (\delta)}{\Omega_{1} \mathrm{~b}_{1} \frac{1}{\frac{1}{\Omega_{2} \mathrm{c}_{1}} \frac{F+s}{s^{\beta}\left(1-\alpha s^{-\alpha}\right)^{\gamma}}-\frac{s+M}{\Omega_{1} \mathrm{~b}_{1}}}}\left(\frac{e^{-y \sqrt{\frac{s+M}{\Omega_{1} \mathrm{~b}_{1}}}}}{s}\right. \\
\left.-\frac{e^{-y \sqrt{\frac{1}{\Omega_{2} \mathrm{c}_{15} \beta\left(1-\alpha s^{-\alpha}\right)^{\gamma}}}}}{s}\right)
\end{gathered}
$$

To find out the Laplace inverse, different authors have used different numerical inverse methods. Therefore, here we will also use the Stehfest algorithm to analyze the solution of temperature and velocity profile numerically. Gaver-Stehfest algorithm [35] mathematically can be defined as

$$
w(\xi, t)=\frac{\ln (2)}{t} \sum_{n=1}^{N} v_{n} \bar{w}\left(\xi, n \frac{\ln (2)}{t}\right)
$$

Where $N$ is a positive integer, and

$$
v_{n}=(-1)^{n+\frac{N}{2}} \sum_{r=\left[\frac{q+1}{2}\right]}^{\min \left(q, \frac{N}{2}\right)} \frac{r^{\frac{N}{2}}(2 r) !}{\left(\frac{N}{2}-r\right) ! r !(r-1) !(q-r) !(2 r-q) !}
$$

However, we have also used another approximation for the solution of temperature and velocity field, Tzou's algorithm for the validity and comparison of our attained numerical results by the Stehfest method. Tzou's method can be defined as mathematically 


$$
w(\xi, t)=\frac{e^{4.7}}{t}\left[\frac{1}{2} \bar{w}\left(r, \frac{4.7}{t}\right)+\operatorname{Re}\left\{\sum_{j=1}^{N}(-1)^{k} \bar{w}\left(r, \frac{4.7+k \pi i}{t}\right)\right\}\right]
$$

Where $i$ is the imaginary unit and $\operatorname{Re}($.) is the real part and $N>1$ is a natural number.

\section{Results and Discussion}

In this article, an unsteady viscous and incompressible MHD fluid flow on an inclined infinite plate with temperature is considered. The non-dimensional leading equations are transformed into a fractional model with help of a new mathematical fractional model namely as Prabhakar fractional derivative. The solution of the transformed fractional model is obtained with the help of LT and different inverse numerical techniques are utilized for the Laplace inverse of governing equations. For the illustration of different parameters on velocity and temperature profiles, figures (2-7) are plotted. In Fig. 2(a-c) the effects of fractional constraint on the temperature profile are highlighted by changing the value of the fractional parameter at different values of the time. This can be seen that the variation in fractional constraint the temperature profile decreases, whereas temperature decreasing away from the plate and asymptotically increases along $y$-direction, which is also referring to the corresponding conditions. Similarly, the behavior of the fractional constraint on the velocity field is displayed in Fig 3(a-c) at different times. This can be seen that in the figure that the velocity profile also decreases with the enhancement in the fractional parameter where with the passage of time velocity field asymptotically increases. In Fig. 4(a-c), the effects of the Grashof number on velocity profile are observed by varying the values of the Grashof number and keeping other values constant. It is observed that enhancement in the values of Grashof number speeds up the motion of the fluid. Physically, an enhancement in Grashof number increases the bouncy forces which rises the thickness of the momentum boundary layer and speed up the fluid motion. Figs. 5 and 6(a-c) highlighting the influence of the angle of inclination of the Magnetic field and the effects of magnetic field parameter on the velocity profile. The velocity field was found to decreases by increasing the values of both parameters, the angle of inclination of magnetic field and magnetic parameter. Physically, an increase in the magnetic field resultsin to increase in Lorentz force which decreases the velocity of the fluid.

The comparison of the numerical techniques namely Stehfest and Tzou'sfor temperature and velocity field is plotted in Fig 7(a) and 7(b) respectively. As plotted in the figures, the curves of both numerical techniquesoverlap with each other for both temperature and velocity field, which represents the validation of our obtained results. Finally, in the end, the numerical 
analysis of the temperature and velocity profile is analyzed in Table 1 different numerical schemes.

\section{Conclusions}

In this work, the solution of unsteady and incompressible fluid flowing on an inclined plate is solved by the mathematical fractional scheme namely as Prabhakar-like thermal fractional technique. The non-dimensional fractional leading equations are solved with help of the LT scheme and different numerical techniques are utilized for the Laplace inverse of temperature and velocity profiles. The influence of different constraints on leading equations is examined graphically and numerically. The main points confabulated from graphical and numerical representation can be bullets as

- The temperature field decreases by raising thevalues of the fractional parameters and asymptotically increases with time.

- The momentum field also decreases with the increase in the values of fractional constraint.

- Velocity profile speeds up by enhancing the value ofGrashof number $G r$ due to increases in buoyancy effect.

- Temperature and velocity profiles both asymptotically increases with the passage of time.

- The solution curves of both numerical techniques overlap with each other which validates our obtained results.

Declaration Statements: The authors declare that they have no known competing financial interests or personal relationships that could have appeared to influence the work reported in this paper.

Availability of data and material: All data that support the findings of this study are included within the article (and any supplementary files).

Competing interests: The authors declared that they have no conflict of interest and the paper presents their own work which does not been infringe any third-party rights, especially authorship of any part of the article is an original contribution, not published before and not being under consideration for publication elsewhere.

Funding: Not applicable.

Authors' contributions: AR and SUK performed the mathematical modeling, AUH and MIK derived the numerical results and MIK draft the final manuscript. 
Acknowledgements: Not applicable.
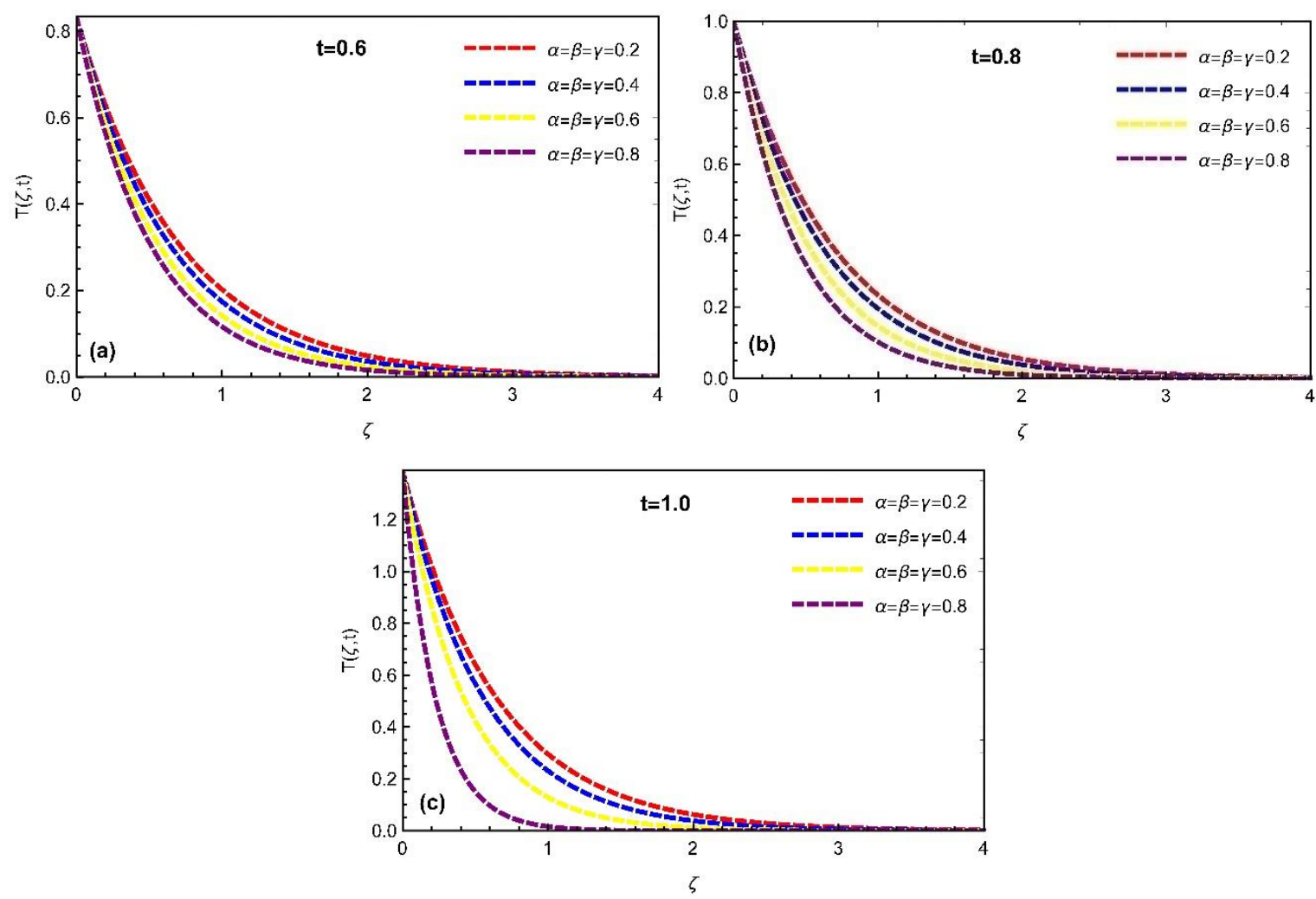

Fig 2: Temperature profile with the variation in fractional parameters at different time
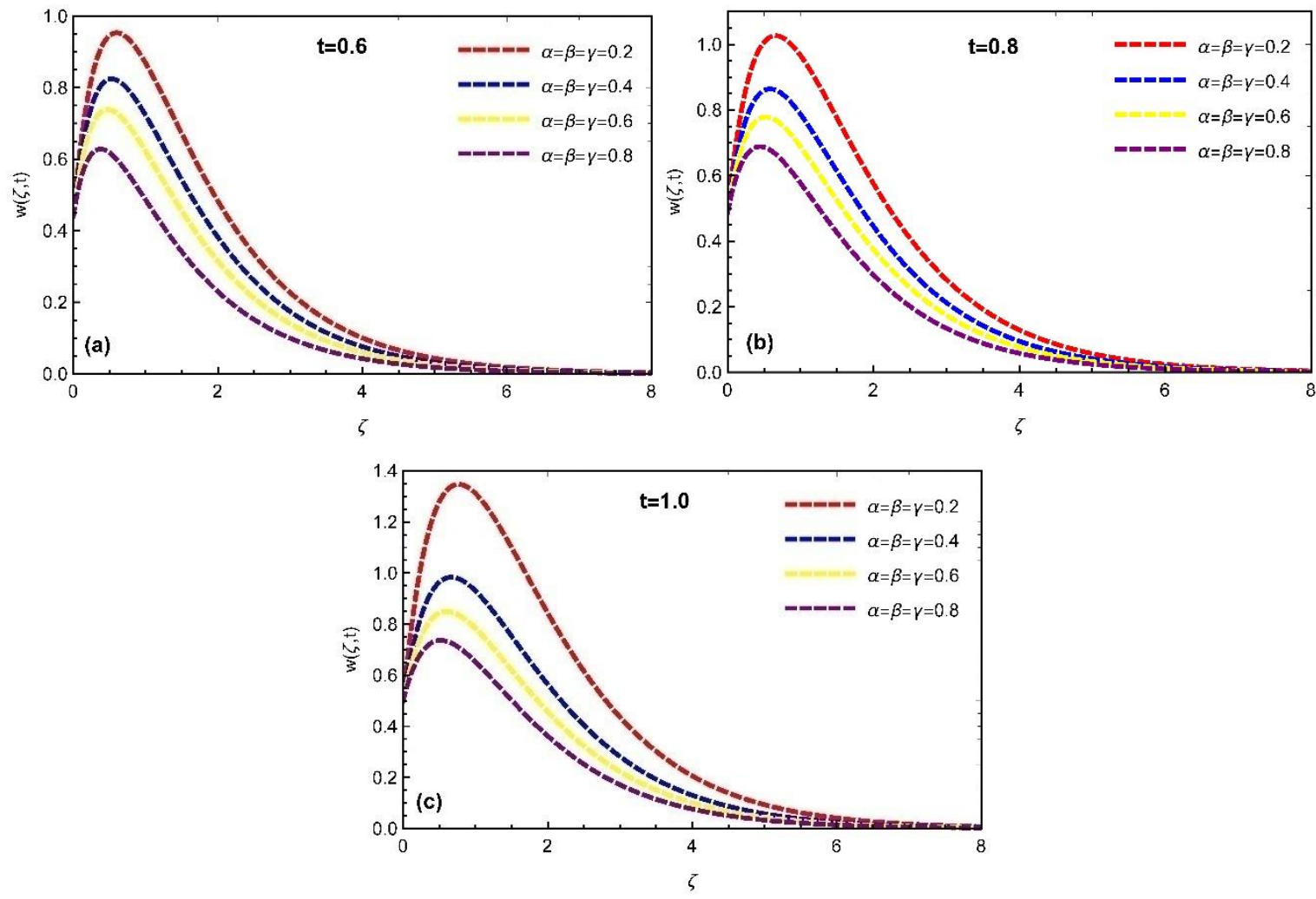
Fig 3: Velocity profile with the variation in fractional constraints $\operatorname{Pr}=6.5, \mathrm{Gr}=$ $0.75, \quad M=0.5, \omega=0.5, \quad \delta=\frac{\pi}{4}, \quad \theta=\frac{\pi}{4}$
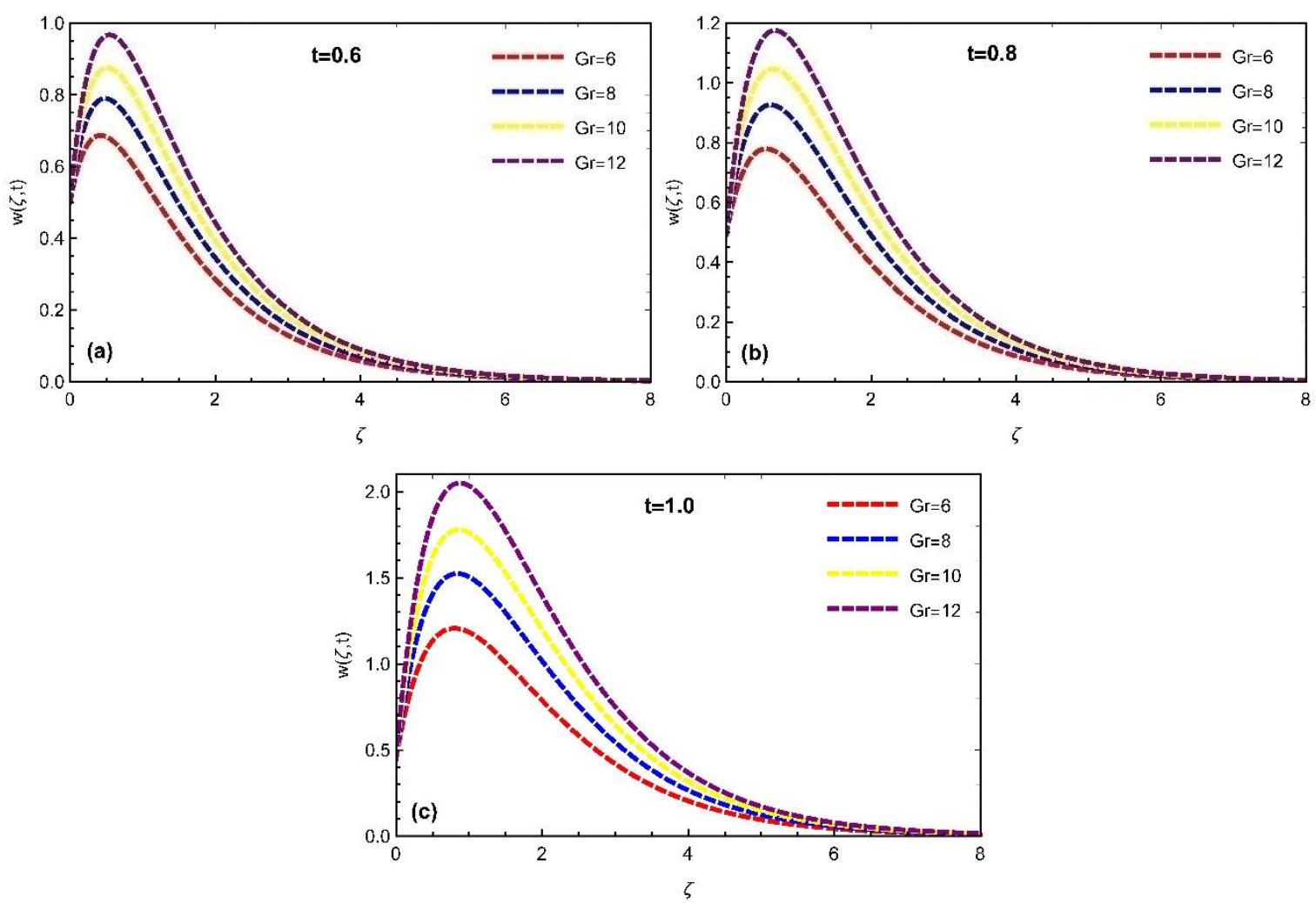

Fig 4: Velocity profile with the variation in Grashof number $\alpha=\beta=\gamma=0.5, \operatorname{Pr}=$

$$
\text { 6.5, } \quad M=0.5, \omega=0.5, \quad \delta=\frac{\pi}{4}, \quad \theta=\frac{\pi}{4}
$$
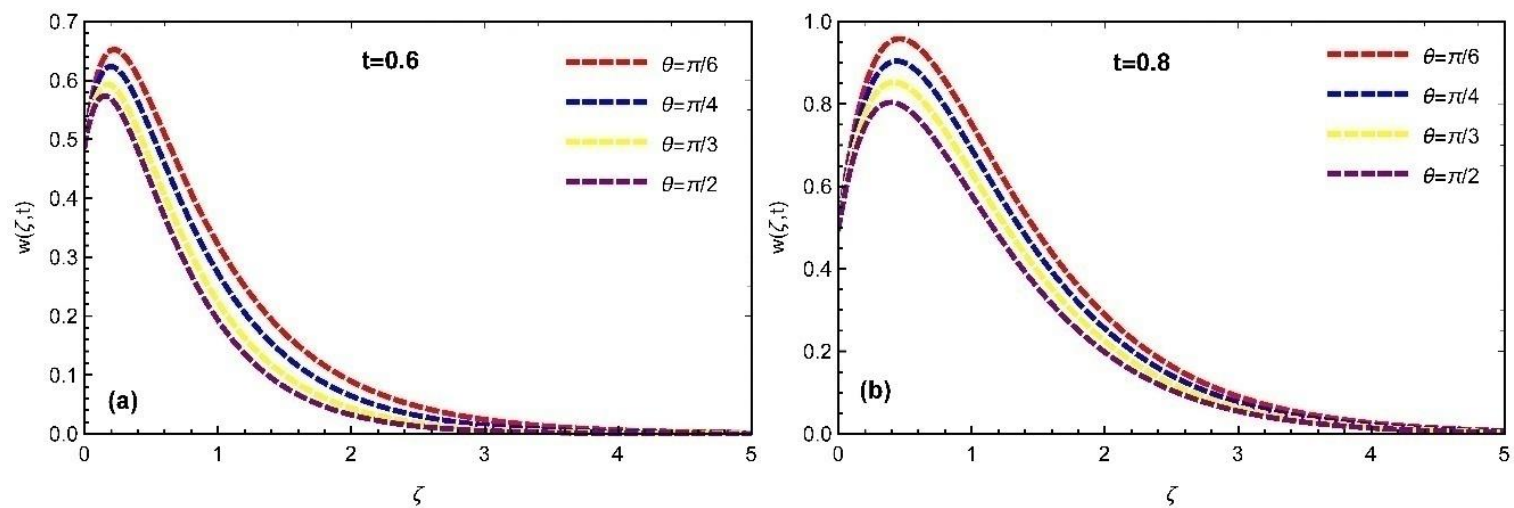


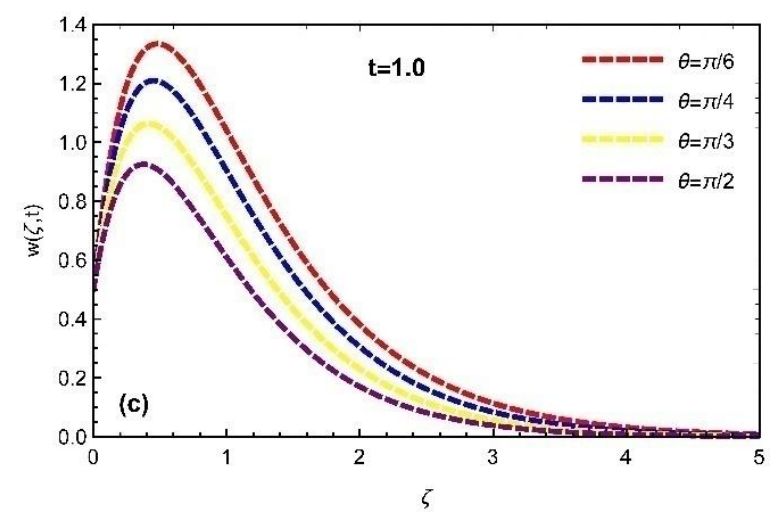

Fig 5: Velocity profile with the variation in the angle of inclination of magnetic field $\alpha=$ $\beta=\gamma=0.5, \operatorname{Pr}=6.5, G r=0.75, \quad M=0.5, \omega=0.5, \quad \delta=\frac{\pi}{4}, \quad \theta=\frac{\pi}{4}$
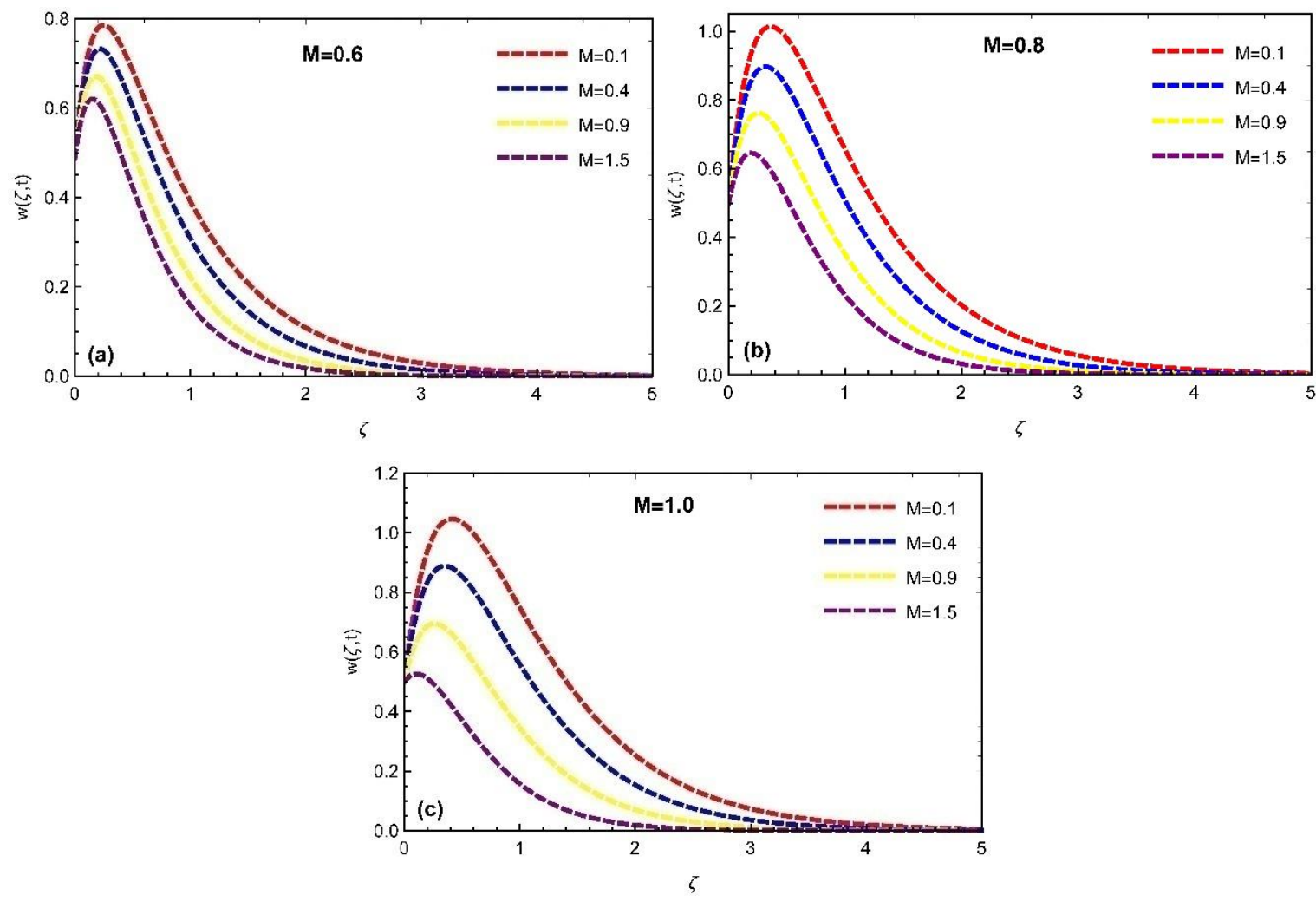

Fig 6: Velocity profile with the variation in Magnetic field $\alpha=\beta=\gamma=0.5, \operatorname{Pr}=6.5$,

$$
G r=0.75, \quad \omega=0.5, \quad \delta=\frac{\pi}{4}, \quad \theta=\frac{\pi}{4}
$$
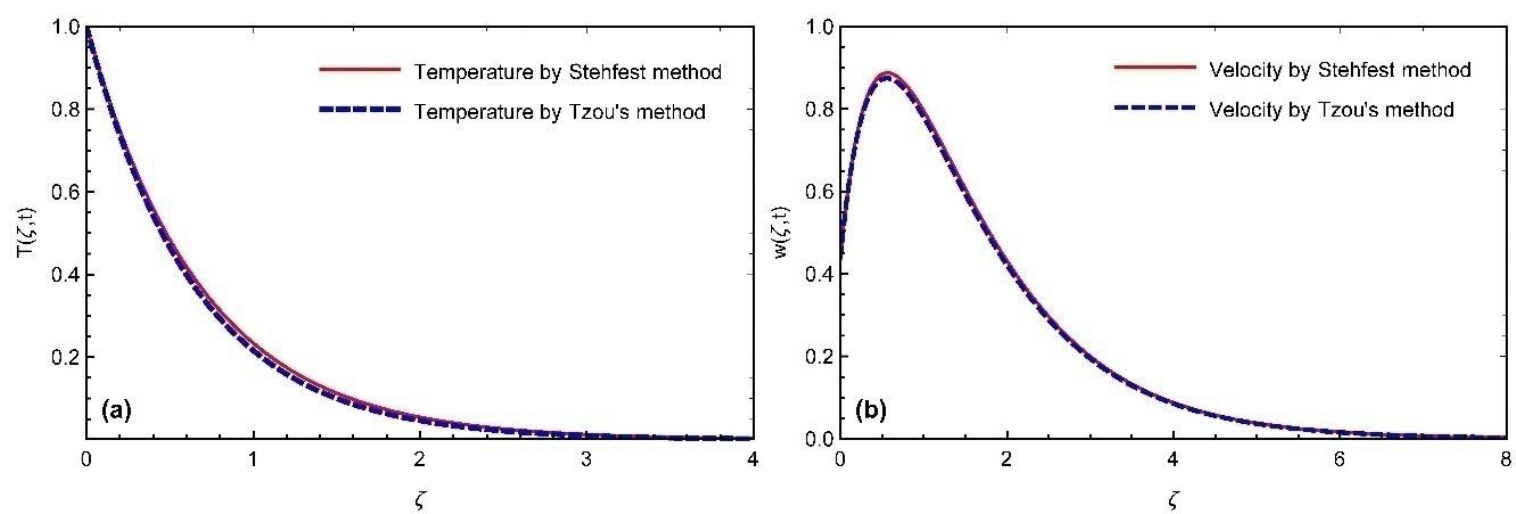
Fig 7: Velocity and temperature profiles comparison for numerical techniques

Table 1: Numerical analysis of temperature and velocity profile by Stehfest and Tzou's method

\begin{tabular}{|c|c|c|c|c|}
\hline$\zeta$ & $\begin{array}{c}\boldsymbol{T}_{(\zeta, t)} \mathbf{b y} \\
\text { Stehfest }\end{array}$ & $\begin{array}{c}\boldsymbol{T}_{(\zeta, t)} \mathbf{b y} \\
\text { Tzou's }\end{array}$ & $\begin{array}{c}\boldsymbol{w}_{(\zeta, \boldsymbol{t})} \mathbf{b y} \\
\text { Stehfest }\end{array}$ & $\begin{array}{c}\boldsymbol{w}_{(\zeta, \boldsymbol{t})} \mathbf{b y} \\
\text { Tzou's }\end{array}$ \\
\hline 0.1 & 0.9763 & 0.9862 & 0.6177 & 0.6172 \\
\hline 0.3 & 0.6635 & 0.6871 & 0.7328 & 0.7312 \\
\hline 0.5 & 0.4444 & 0.4629 & 0.7793 & 0.7770 \\
\hline 0.7 & 0.2931 & 0.3072 & 0.7802 & 0.7775 \\
\hline 0.9 & 0.1903 & 0.2009 & 0.7516 & 0.7487 \\
\hline 1.1 & 0.1217 & 0.1294 & 0.7051 & 0.7022 \\
\hline 1.3 & 0.0766 & 0.0821 & 0.6489 & 0.6460 \\
\hline 1.5 & 0.0475 & 0.0513 & 0.5885 & 0.5858 \\
\hline 1.7 & 0.0290 & 0.0316 & 0.5278 & 0.5253 \\
\hline 1.9 & 0.0174 & 0.0192 & 0.4690 & 0.4667 \\
\hline
\end{tabular}

\section{References}

[1].M.Turkyilmazoglu, Three dimensional MHD flow and heat transfer over a stretching/shrinking surface in a viscoelastic fluid with various physical effects, International Journal of Heat and Mass Transfer, Volume 78, November 2014, Pages $150-155$

[2].S.R.Mishra, M.M.Bhatti, Simultaneous effects of chemical reaction and Ohmic heating with heat and mass transfer over a stretching surface: A numerical study, Chinese Journal of Chemical Engineering, Volume 25, Issue 9, September 2017, Pages 11371142

[3].Manoj KumarNayak, Gauranga Charan Dash, Lambodar Prased Singh, Heat and mass transfer effects on MHD viscoelastic fluid over a stretching sheet through porous 
medium in presence of chemical reaction, Propulsion and Power Research, Volume 5, Issue 1, March 2016, Pages 70-80

[4].Sultan Z.Alamri, Ambreen A.Khan, Mariam Azeez, R.Ellahi, Effects of mass transfer on MHD second grade fluid towards stretching cylinder: A novel perspective of Cattaneo-Christov heat flux model, Physics Letters A, Volume 383, Issues 2-3, 12 January 2019, Pages 276-281

[5].Jing Li, Liancun Zheng, Lin Liu, MHD viscoelastic flow and heat transfer over a vertical stretching sheet with Cattaneo-Christov heat flux effects, Journal of Molecular Liquids, Volume 221, September 2016, Pages 19-25

[6].Iskandar Waini, Anuar Ishak, Ioan Pop, Transpiration effects on hybrid nanofluid flow and heat transfer over a stretching/shrinking sheet with uniform shear flow, Alexandria Engineering Journal, Volume 59, Issue 1, February 2020, Pages 91-99

[7]. Tasawar Hayat, Muhammad Ijaz Khan, Ahmad Alsaedi, Muhammad Imran Khan, Homogeneous-heterogeneous reactions and melting heat transfer effects in the MHD flow by a stretching surface with variable thickness, Journal of Molecular Liquids, Volume 223, November 2016, Pages 960-968

[8]. Mohsan Hassan, Essam R El-Zahar, Sami Ullah Khan, Mohammad Rahimi-Gorji, Ashfaq Ahmad, Boundary layer flow pattern of heat and mass for homogenous shear thinning hybrid-nanofluid: An experimental data base modeling, Numerical Methods for Partial Differential Equations, Volume 37, Issue2, March 2021, pp. 1234-1249

[9].L.T.Benos, K.R.Nagaraju, U.S.Mahabaleshwar, M.S.Prasad, I.E.Sarris, G.Lorenzini, Magnetohydrodynamic and radiation effects on the heat transfer of a continuously stretching/shrinking sheet with mass transpiration of the horizontal boundary, Chinese Journal of Physics, Volume 72, August 2021, Pages 700-715.

[10]. B. Mahanthesh, S. A. Shehzad, T. Ambreen \& S. U. Khan, Significance of Joule heating and viscous heating on heat transport of $\mathrm{MoS}_{2}-\mathrm{Ag}$ hybrid nanofluid past an isothermal wedge, Journal of Thermal Analysis and Calorimetry, volume 143, pp. 1221-1229 (2021).

[11]. Faisal Shahzad, Wasim Jamshed, Kottakkaran Sooppy Nisar, M. Motawi Khashan, Abdel-Haleem Abdel-Aty, Computational analysis of Ohmic and viscous dissipation effects on MHD heat transfer flow of $\mathrm{Cu}$-PVA Jeffrey nanofluid through a stretchable surface, Case Studies in Thermal Engineering, Volume 26, August 2021, 101148 
[12]. M. Ijaz Khan, Hassan Waqas, Sami Ullah Khan, Muhammad Imran, Yu-Ming Chu, Aamar Abbasi, Seifedine Kadry, Slip flow of micropolar nanofluid over a porous rotating disk with motile microorganisms, nonlinear thermal radiation and activation energy, International Communications in Heat and Mass Transfer, Volume 122, March (2021), 105161.

[13]. Caputo M., Fabrizio M.A new definition of fractional derivative without singular kernel, Progr Fract Differ Appl, 1 (2015), pp. 73-85

[14]. Nesrine Harrouche, Shaher Momani, Shatha Hasan, Mohammed Al-Smadi, Computational algorithm for solving drug pharmacokinetic model under uncertainty with nonsingular kernel type Caputo-Fabrizio fractional derivative, Alexandria Engineering Journal, Volume 60, Issue 5, October 2021, Pages 4347-4362

[15]. Elvin J. Moore, Sekson Sirisubtawee \& Sanoe Koonprasert ,A Caputo-Fabrizio fractional differential equation model for HIV/AIDS with treatment compartment, Advances in Difference Equations volume 2019, Article number: 200 (2019).

[16]. Leilei Wei, Wenbo $\mathrm{Li}$, Local discontinuous Galerkin approximations to variable-order time-fractional diffusion model based on the Caputo-Fabrizio fractional derivative, Mathematics and Computers in Simulation, Volume 188, October 2021, Pages 280-290

[17]. Ebraheem Alzahrani, M.M.El-Dessoky, Dumitru Baleanu, Modeling the dynamics of the novel coronavirus using Caputo-Fabrizio derivative, Alexandria Engineering Journal, Volume 60, Issue 5, October 2021, Pages 4651-4662

[18]. Shaher Momani, Nadir Djeddi, Mohammed Al-Smadi, Shrideh Al-Omari, Numerical investigation for Caputo-Fabrizio fractional Riccati and Bernoulli equations using iterative reproducing kernel method, Applied Numerical Mathematics, Volume 170, December 2021, Pages 418-434

[19]. Amjad Shaikh, Asifa Tassaddiq, Kottakkaran Sooppy Nisar and Dumitru Baleanu, Analysis of differential equations involving Caputo-Fabrizio fractional operator and its applications to reaction-diffusion equations, Advances in Difference Equations, 2019) 2019:178

[20]. Xiaobin Wang, Muhammad Shoaib Saleem, Kiran Naseem Aslam, Xingxing $\mathrm{Wu}$, and Tong Zhou, On Caputo-Fabrizio Fractional Integral Inequalities of HermiteHadamard Type for Modified -Convex Functions, Journal of Mathematics, Volume 2020, Article ID 8829140. 
[21]. Dumitru Baleanu, Hakimeh Mohammadi \& Shahram Rezapour, A fractional differential equation model for the COVID-19 transmission by using the CaputoFabrizio derivative, Advances in Difference Equations volume 2020, Article number: 299 (2020)

[22]. Atangana A., Baleanu D. New fractional derivatives with non-local and nonsingular kernel: Theory and application to heat transfer model, Thermal Sci, 20 (2016), pp. $763-769$

[23]. N.A. Sheikh, F. Ali, M. Saqib, I. Khan, S.A.A. Jan, A.S. Alshomrani, M.S. Alghamdi, Comparison and analysis of the Atangana-Baleanu and Caputo-Fabrizio fractional derivatives for generalized Casson fluid model with heat generation and chemical reaction, Results Phys., 7 (2017), pp. 789-800

[24]. Panda S.K., Ravichandran C., Hazarika B. Results on system of AtanganaBaleanu fractional order Willis aneurysm and nonlinear singularly perturbed boundary value problems, Chaos Solitons Fractal, 142 (2021), Article 110390

[25]. Pushpendra Kumar, Vedat Suat Erturk, Hassan Almusawa, Mathematical structure of mosaic disease using microbial biostimulants via Caputo and AtanganaBaleanu derivatives, Results in Physics, Volume 24, May 2021, 104186.

[26]. Ali Raza, Sami Ullah Khan, Saadia Farid, M. Ijaz Khan, Tian-Chuan Sun, Aamar Abbasi,M. Imran Khan, M. Y. Malik, Thermal activity of conventional Casson nanoparticles with ramped temperature due to an infinite vertical plate via fractional derivative approach, Case Studies in Thermal Engineering, Volume 27, October 2021, 101191 .

[27]. M.Mallika Arjunan, A.Hamiaz, V.Kavitha, Existence results for AtanganaBaleanu fractional neutral integro-differential systems with infinite delay through sectorial operators, Chaos, Solitons \& Fractals, Volume 149, August 2021, 111042.

[28]. Pushpendra Kumar, Vedat SuatErturk, HassanAlmusawa, Mathematical structure of mosaic disease using microbial biostimulants via Caputo and AtanganaBaleanu derivatives, Results in Physics, Volume 24, May 2021, 104186.

[29]. Xiao-Ping Li, Nadia Gul, Muhammad Altaf Khan, Rubi Bilal, Aatif Ali, Mohammad Y.Alshahrani, Taseer Muhammad, SaeedIslam, A new Hepatitis B model in light of asymptomatic carriers and vaccination study through Atangana-Baleanu derivative, Results in Physics, Volume 29, October 2021, 104603.

[30]. Ying-Qing Song Ali Raza, Kamel Al-Khaled, Saadia Farid, M. Ijaz Khan, Sami Ullah Khan, Qiu-HongShi, M.Y.Malik, M. Imran Khan, Significances of exponential 
heating and Darcy's law for second grade fluid flow over oscillating plate by using Atangana-Baleanu fractional derivatives, Case Studies in Thermal Engineering, Volume 27, October 2021, 101266.

[31]. Mohammad Partohaghighi, Ali Akgül, Modelling and simulations of the SEIR and Blood Coagulation systems using Atangana-Baleanu-Caputo derivative, Chaos, Solitons \& Fractals, Volume 150, September 2021, 111135.

[32]. Bing Guo, Ali Raza, Kamel Al-Khaled, Sami Ullah Khan, Saadia Farid, Ye Wang, M. Ijaz Khan, M. Y. Malik, S. Saleem, Fractional-order simulations for heat and mass transfer analysis confined by elliptic inclined plate with slip effects: A comparative fractional analysis, Case Studies in Thermal Engineering, Volume 28, December 2021, 101359

[33]. A. Giusti and I. Colombaro, "Prabhakar-like fractional viscoelasticity," Communications in Nonlinear Science and Numerical Simulation, vol. 56, pp. 138-143, 2018

[34]. F. Polito and Z. Tomovski, "Some properties of Prabhakar-type fractional calculus operators," arXiv preprint arXiv:1508.03224, 2015.

[35]. H. Stehfest, "Algorithm 368: Numerical inversion of Laplace transforms [D5]," Communications of the ACM, vol. 13, no. 1, pp. 47-49, 1970 